www.nature.com/pj

\title{
Fabrication of a poly(vinyl alcohol) monolith via thermally impacted non-solvent-induced phase separation
}

\author{
Xiaoxia Sun, Takashi Fujimoto and Hiroshi Uyama
}

\begin{abstract}
A monolith of poly(vinyl alcohol) (PVA) was successfully prepared by thermally impacted non-solvent-induced phase separation without any templates. The morphology of the PVA monolith was observed by scanning electron microscopy. The pore and skeleton sizes could be controlled by changing the cooling temperature as well as the concentration and molecular weight of PVA. The nitrogen adsorption-desorption isotherms showed that the PVA monolith has a large surface area and uniform mesopore structure. Crosslinking using a glutaraldehyde (GA) agent produced a water-insoluble monolith. By changing the concentration of GA, a crosslinked monolith with various swelling ratios for water was obtained.

Polymer Journal (2013) 45, 1101-1106; doi:10.1038/pj.2013.18; published online 6 March 2013
\end{abstract}

Keywords: crosslinking; monolith; non-solvent phase separation; poly(vinyl alcohol)

\section{INTRODUCTION}

In general, 'monolith' means 'one piece'. In the field of chromatography, this term is used in contrast to a particulate stationary phase, which is defined as porous materials that have a three-dimensional continuous interconnected pore structure. ${ }^{1,2}$ In the last two decades, considerable attention has been given to monoliths because of their excellent permeability, fast mass transfer performance, high stability and ease of chemical modification. ${ }^{3-5}$ On the basis of these favorable properties, monolithic materials can be used for chromatography, ${ }^{6-8}$ oil removal, ${ }^{9}$ biomolecule immobilization, ${ }^{10}$ controlled drug release ${ }^{11}$ and supported catalysis. ${ }^{12-15}$

Monoliths can be divided into polymer-based and silica-based types. ${ }^{16,17}$ Compared with silica-based monoliths, which have some innate defects such as complicated and sensitive production processes and limited $\mathrm{pH}$ tolerance, ${ }^{18}$ porous polymer monoliths have received increased interest not only for their predominant $\mathrm{pH}$ stability, nonspecific interaction and biocompatibility ${ }^{19-21}$ but also for their specific pore properties and easy functionalization. ${ }^{22-24}$

To date, significant progress has been made in monolith preparation technology. There is a large variety of methods for producing porous polymer monoliths from many types of monomers. The first method used to fabricate polymer-based monoliths is thermally initiated free radical polymerization in an unstirred mold. ${ }^{25-28}$ Radiation polymerizations initiated by high energy radiation such as $\gamma$-rays ${ }^{29}$ or electron beams, ${ }^{30}$ a polymerized high internal phase emulsion method, ${ }^{31}$ crygels, $^{32}$ living polymerization ${ }^{33}$ and polycondensation reactions ${ }^{34}$ have also been used to fabricate polymeric monoliths. ${ }^{28}$ For all the reported methods, a monomer is an indispensable component because it acts as a precursor for polymerization, in which phase separation takes place to form the monolith. In most cases, however, complicated and time-consuming fabrication processes, including the use of other reagents such as crosslinkers and porogen, are involved. In addition, the simultaneous precise control of polymerization and phase separation is required.

Recently, we have reported a novel approach to fabricate a polymer-based monolith using a polymer, rather than a monomer, by the thermally induced phase separation technique. ${ }^{35,36}$ For the polyacrylonitrile monolith, polyacrylonitrile is first dissolved into a mixture of solvent and non-solvent by heating, followed by cooling to room temperature. During this cooling process, the phase separation forms the mesoporous polyacrylonitrile monolith without any templates. By this method, the shape of the monolith can be easily designed for various applications by altering the vessel shape.

In this study, a new class of methods for fabricating monoliths, named thermally impacted non-solvent-induced phase separation (TINIPS), is proposed. This method is based on non-solvent-induced phase separation, which is usually applied to fabricate polymer membranes; ${ }^{37,38}$ here, we introduce a thermal factor into nonsolvent-induced phase separation. The TINIPS method is a remarkably simple and clean process that involves the addition of non-solvent to the polymer solution at high temperature and the subsequent cooling of the mixed solution, which contains a polymer, solvent and non-solvent. In the cooling process, the phase separation forms the monolithic material. Moreover, this method is template free; thus, it enables the convenient fabrication of functional monoliths, satisfying the demand for a great variety of shapes. 
Poly(vinyl alcohol) (PVA) is one of the most typical hydrophilic and water-soluble polymers that is produced by the polymerization of vinyl acetate, followed by alkaline hydrolysis. ${ }^{39,40}$ PVA is an outstanding biomaterial with several advantages, such as biocompatibility and nontoxicity, ${ }^{40,41}$ which make it suitable for versatile applications, for example, wound dressings, ${ }^{42}$ antibacterial materials, ${ }^{43}$ soft tissue replacement ${ }^{44}$ and the immobilization of microorganisms. ${ }^{45}$ This study addresses the first fabrication of a PVA monolith via the TINIPS method. The gross shape and internal structure of the PVA monolith can be controlled precisely by adjusting the fabrication parameters. A convenient fabrication method for hydrophilic porous polymer monoliths will be significant in the development of monolith applications in biomedical fields.

\section{EXPERIMENTAL PROCEDURE}

\section{Materials}

PVA powders with a hydrolysis ratio of $98 \%$ and different molecular weights were purchased from Wako Pure Chemical Industries, Ltd (Tokyo, Japan), and Sigma-Aldrich Co. (Tokyo, Japan). Glutaraldehyde (GA) solution (50 wt\%) was obtained from Sigma-Aldrich Co. (Tokyo, Japan). Hydrochloric acid $\left(1 \mathrm{moll}^{-1}\right)$ was purchased from Wako Pure Chemical Industries, Ltd. All other reagents and solvents were used as received.

\section{Measurements}

The microstructure of PVA monolith was observed with a scanning electron microscope (SEM, S-3000N, Hitachi Ltd, Tokyo, Japan) at $15 \mathrm{kV}$. Before observation, the sample was cut with a scalpel and coated with a thin layer of gold by an ion sputter apparatus (E-1010 Ion Sputter, Hitachi Ltd). The specific surface area of the samples was measured by a nitrogen adsorption apparatus using the Brunauer-Emmett-Teller method (NOVA 4200e, Quantachrome Instruments, Boynton Beach, FL, USA). PVA monoliths before and after crosslinking were analyzed using a Nicolet iS 5 Fourier transform infrared spectrophotometer (Thermo Scientific, Yokohama, Japan). X-ray diffraction patterns were obtained by an XRD-6100 instrument (Sigmadzu Corp., Kyoto, Japan) at a scanning rate of $4.0 \mathrm{deg} \mathrm{min}^{-1}$ from $10^{\circ}$ to $70^{\circ}(2 \theta)$. The measurement was performed under conditions of $40 \mathrm{kV}$ and $30 \mathrm{~mA}$ using $\mathrm{Cu}-\mathrm{K} \alpha$ radiation $(\lambda=1.5418 \AA)$.

\section{Preparation of PVA monolith}

PVA powder was first dissolved into distilled water at $95^{\circ} \mathrm{C}$ for approximately $3 \mathrm{~h}$ with constant stirring at 400 r.p.m. to form a homogeneous aqueous PVA solution. The solution was cooled to $55^{\circ} \mathrm{C}$, and then acetone (non-solvent) was added dropwise to avoid the formation of precipitates. Then, the mixture was maintained at $20^{\circ} \mathrm{C}$ for $24 \mathrm{~h}$, during which the phase separation took place, forming a white monolithic material. The solvent in the monolith was replaced by acetone by immersion of the monolith into $60 \mathrm{ml}$ of acetone for $12 \mathrm{~h}$ under gentle shaking. The solvent replacement was repeated three times, and the monolith was finally dried in vacuo.

\section{Crosslinking of PVA monolith by GA}

The monolith $(210 \mathrm{mg}$ ) was immersed in $15 \mathrm{ml}$ of the GA solution with $0.3 \mathrm{ml}$ of $1 \mathrm{M} \mathrm{HCl}$ as catalyst for various lengths of time. The monolith was washed with distilled water until the water became neutral and then was dried in vacuo for $12 \mathrm{~h}$.

\section{Determination of swelling ratio of PVA monolith}

The PVA monolith (approximately $210 \mathrm{mg}$ ) was immersed in $20 \mathrm{ml}$ of water at room temperature. The weight change was measured every $5 \mathrm{~min}$ until the weight became nearly constant. The experiment was carried out three times. The swelling ratio was calculated as follows: ${ }^{46}$

$$
\text { swelling ratio }(\%)=\left(W_{\mathrm{b}}-W_{\mathrm{e}}\right) / W_{\mathrm{e}} \times 100
$$

where $W_{\mathrm{e}}$ and $W_{\mathrm{b}}$ are the weights before and after the immersion in water, respectively.

\section{RESULTS AND DISCUSSION}

\section{Fabrication and characterizations of PVA monolith}

The PVA monolith is first fabricated from the mixture containing PVA, water and acetone. The fabrication conditions are as follows: a cooling temperature of $20^{\circ} \mathrm{C}$, a PVA concentration of $60 \mathrm{mg} \mathrm{m}^{-1}$, a PVA molecular weight of $8.8 \times 10^{4}$ and a water/acetone mixed ratio of $4 / 3$. The internal morphology of the PVA monolith is observed by SEM, which shows the three-dimensional open pore structures of the monolith (Figure 1). The average pore and skeleton sizes of the PVA monolith are 2.0 and $1.1 \mu \mathrm{m}$, respectively.

The adsorption-desorption isotherm of the PVA monolith is shown in Figure 2a. The isotherm is ascribed to type IV, demonstrating the existence of mesopores in the monolith. The isotherm at the beginning is monolayer adsorption, followed by multilayer adsorption and the hysteresis in the multilayer range is associated with capillary condensation in the mesopore structures. The hysteresis loop in the $\mathrm{P} / \mathrm{P}_{0}$ range from 0.7 to 1.0 is of type $\mathrm{H} 1$, indicating that the PVA monolith contains cylindrical pores with narrow distributions of pore size. ${ }^{47,48}$ The Brunauer-Emmett-Teller surface area is $110 \mathrm{~m}^{2} \mathrm{~g}^{-1}$, and the pore size distribution plot for the sample obtained by using the non-local density functional theory method reveals relatively uniform mesopores with pore diameters of $7-25 \mathrm{~nm}$ (Figure $2 \mathrm{~b}$ ). These results indicate that the PVA monolith is a mesoporous material with a large surface area.

The crystalline structure of the monolith is examined by wide-angle X-ray diffraction (see Supplementary Figure S1). A relatively sharp and intense peak is observed at $2 \theta=19.5^{\circ}$ for the monolith, which is nearly the same as that of the PVA powder. The microcrystallites of the PVA powder were reported to have been formed by the aggregation of hydrogen bonds between the hydroxyl groups. ${ }^{49,50}$
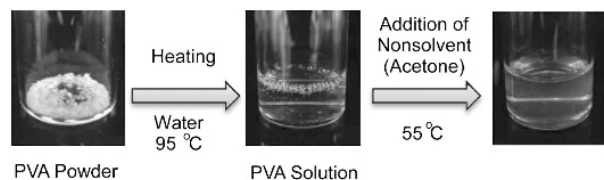

PVA Powder PVA Solution
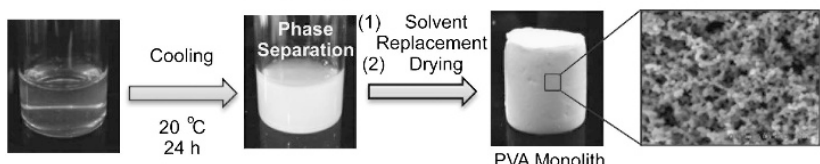

Figure 1 Fabrication processes of the poly(vinyl alcohol) (PVA) monolith.

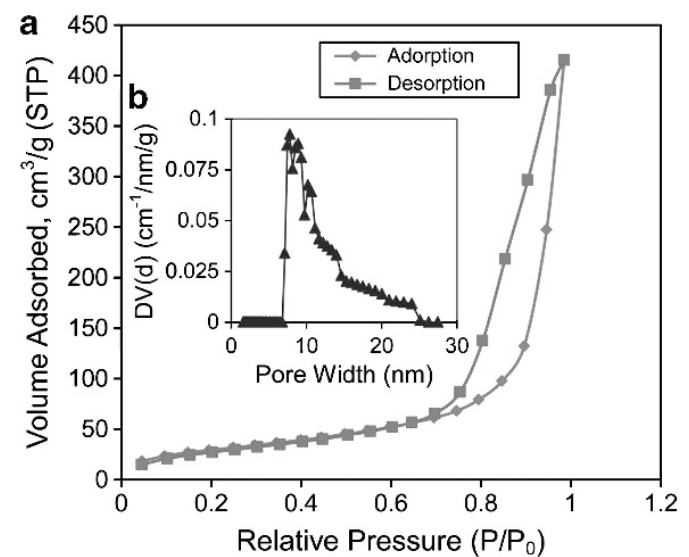

Figure 2 Nitrogen adsorption-desorption isotherms of the poly(vinyl alcohol) (PVA) monolith (a) and the pore size disbribution plot for the PVA monolith (b). A full color version of this figure is available at Polymer Journal online. 

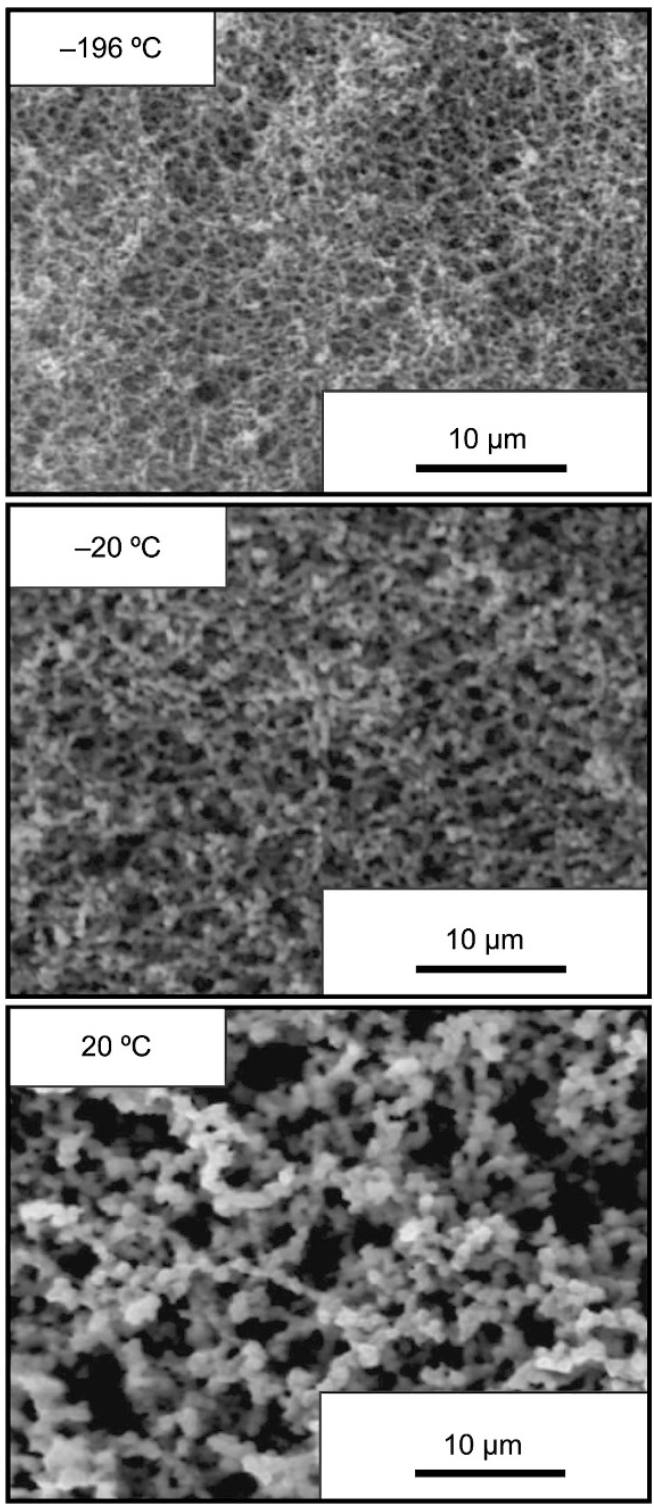

Figure 3 Scanning electron microscopy (SEM) photography of microstructures of the poly(vinyl alcohol) (PVA) monolith fabricated at different cooling temperatures.

The similar results of X-ray diffraction for the monolith and PVA powder strongly suggest that the microcrystallites of the PVA monolith are also configured via hydrogen bonds.

\section{Morphological control of PVA monolith}

The mixed ratio of water and acetone is examined with a cooling temperature of $20^{\circ} \mathrm{C}$, a PVA concentration of $60 \mathrm{mg} \mathrm{ml}^{-1}$ and a PVA molecular weight of $8.8 \times 10^{4}$. As described above, the water/acetone mixed ratio of $4 / 3$ afford the monolith a uniform shape. Conversely, phase separation does not take place for a mixed ratio of $2 / 1$, resulting in no monolith formation. The PVA powder is not completely solubilized for a mixed ratio of $1 / 1$. These results indicate that the mixed ratio of $4 / 3$ is a crucial factor for fabrication of the PVA monolith.

Figure 3 shows SEM photographs of the PVA monolith fabricated with cooling temperatures of $-196,-20$ and $20^{\circ} \mathrm{C}$ with a PVA concentration of $60 \mathrm{mg} \mathrm{ml}^{-1}$, a PVA molecular weight of $8.8 \times 10^{4}$ and

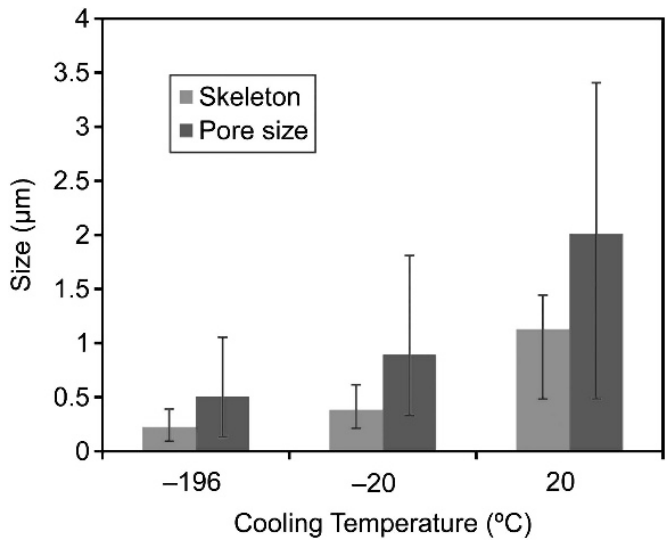

Figure 4 Effect of cooling temperatures on average pore and skeleton sizes. A full color version of this figure is available at Polymer Journal online.

a water/acetone mixed ratio of $4 / 3$. The average skeleton and pore sizes increase with increasing cooling temperature (see Figure 4 and Supplementary Table S1). This increase may occur because, at higher temperatures, PVA molecules can move more freely and have sufficient time to coagulate and form a monolith with larger skeleton and pore sizes, whereas at lower temperatures, the movement of PVA molecules is limited, and the phase separation takes place more quickly, reducing the skeleton and pore sizes. When the cooling temperature is $-196^{\circ} \mathrm{C}$, the mixture freezes instantaneously, resulting in the smallest skeleton and pore sizes observed.

Next, the effect of PVA concentration on the morphology of the monolith is examined. Figure 5 shows SEM photographs of the PVA monolith fabricated with different PVA concentrations with a temperature of $20^{\circ} \mathrm{C}$, a PVA molecular weight of $8.8 \times 10^{4}$ and a water/acetone mixed ratio of $4 / 3$. In all the cases examined, a relatively uniform skeleton is formed. The skeleton and pore sizes decrease as a function of PVA concentration in the range of $40-80 \mathrm{mg} \mathrm{ml}^{-1}$ (Figure 6). These data strongly suggest that the morphology is easily controlled by changing the cooling temperature and PVA concentration. With increasing viscosity of the polymer solution, a higher degree of entanglement results in slower dynamics of the phase separation. Therefore, the increase in the PVA concentration leads to a decrease in the skeleton and pore sizes in the fabrication of the PVA monolith.

In the above experiments, the monolith is fabricated using PVA with a molecular weight of $8.8 \times 10^{4}$. PVAs with different molecular weights, $1.8 \times 10^{4}$ and $1.7 \times 10^{5}$, are also used to examine the effect of the PVA molecular weight on the morphology of the monolith with a cooling temperature of $20^{\circ} \mathrm{C}$, a PVA concentration of $60 \mathrm{mg} \mathrm{ml}^{-1}$ and a water/acetone mixed ratio of $4 / 3$ (Figure 7 ). In all the cases examined, the monolith is formed. When the molecular weight is $1.8 \times 10^{4}$, the skeleton and pore sizes are larger than those with higher molecular weight (Figure 8). The size difference between the molecular weights of $8.8 \times 10^{4}$ and $1.7 \times 10^{5}$ is relatively small. These data clearly show that PVAs with a wide range of molecular weights are applicable for the fabrication of PVA monoliths by the TINIPS method and that the molecular weight affects the morphology of the PVA monolith. The morphology difference may be due to the mobility of the polymer chain in the present system; the domain formation in the phase separation is strongly affected by the viscosity of the polymer solution of PVA, depending on the molecular weight. 

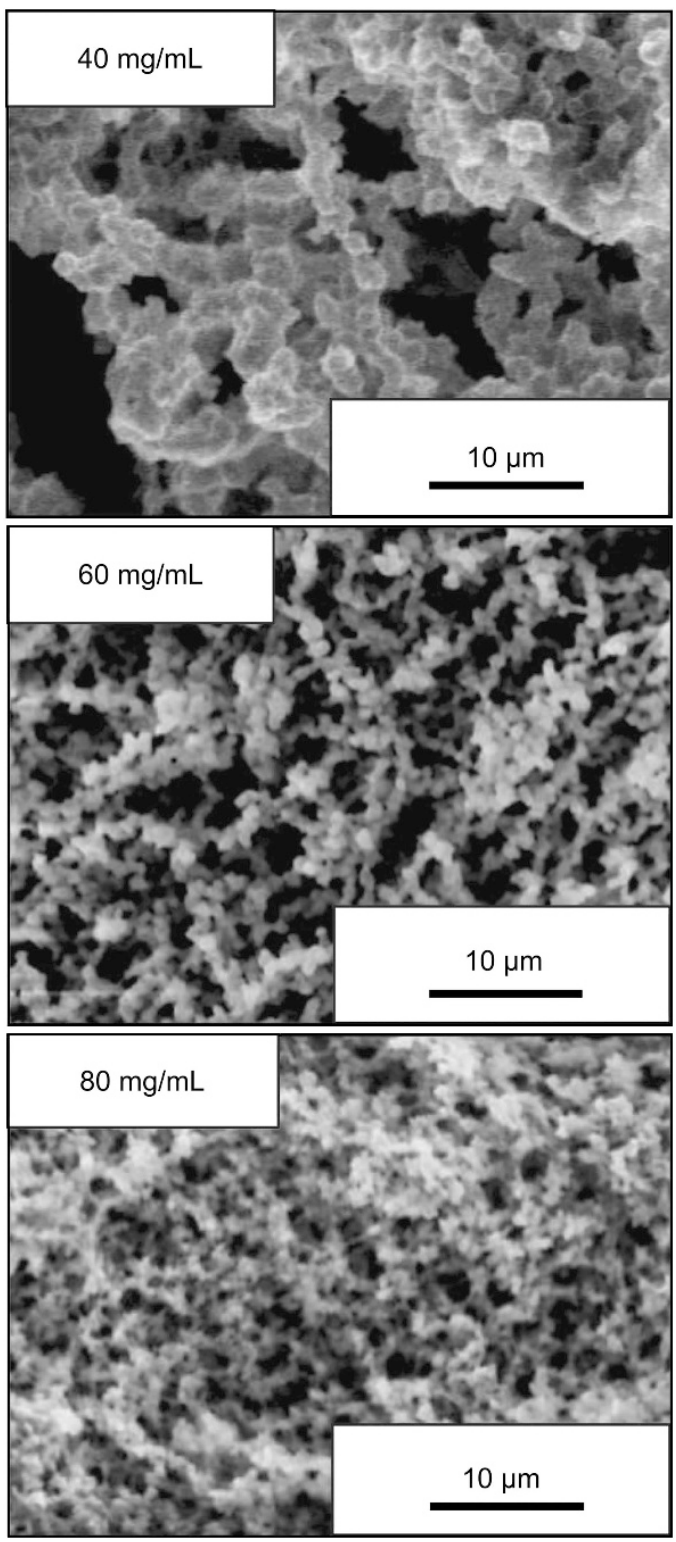

Figure 5 Scanning electron microscopy (SEM) photography of microstructures of the poly(vinyl alcohol) (PVA) monolith fabricated from different concentrations of PVA.

\section{Crosslinking of PVA monolith}

GA is known to be an effective crosslinking agent for PVA. ${ }^{51}$ In this study, GA is selected to crosslink the PVA monolith. The crosslinking is performed by immersion of the monolith in the GA solution with concentrations of $6.25,12.5$ and $25 \mathrm{wt} \%$ for 6,12 or $24 \mathrm{~h}$ in the presence of a small amount of $\mathrm{HCl}$ as catalyst. In all the cases examined, the crosslinking takes place smoothly, forming a waterinsoluble monolith. The crosslinking is confirmed by Fourier transform infrared (see Supplementary Figure S2). In the Fourier transform infrared spectrum of the product after crosslinking, a strong peak at $1720 \mathrm{~cm}^{-1}$ ascribed to the carbonyl group of aldehyde and two peaks at 2850 and $2750 \mathrm{~cm}^{-1}$ because of $\mathrm{C}-\mathrm{H}$ stretching appear. $^{52,53}$ Furthermore, the intensity of the broad peak around $3300 \mathrm{~cm}^{-1}$ because of $\mathrm{O}-\mathrm{H}$ stretching of PVA decreases in comparison with that of PVA. These results suggest that the

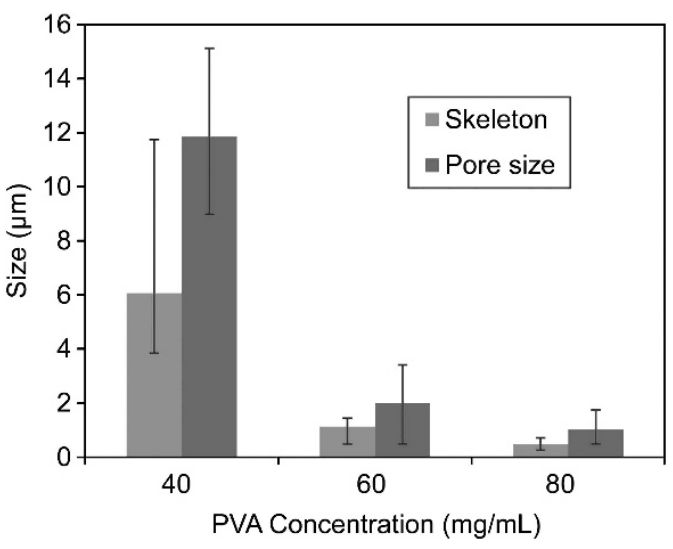

Figure 6 Effect of poly(vinyl alcohol) (PVA) concentration on average pore and skeleton sizes. A full color version of this figure is available at Polymer Journal online.

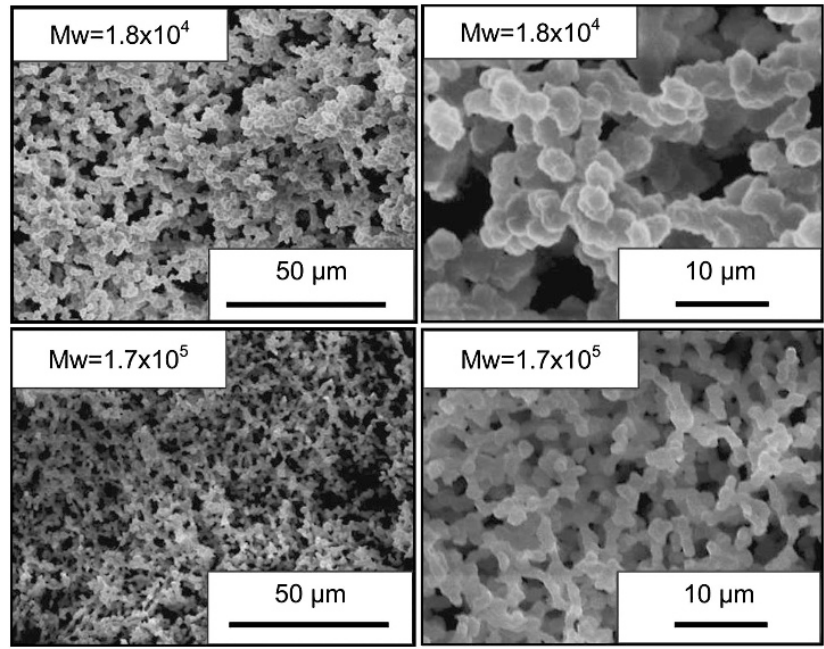

Figure 7 Scanning electron microscopy (SEM) photography of microstructures of the poly(vinyl alcohol) (PVA) monolith fabricated with various PVA molecular weights.

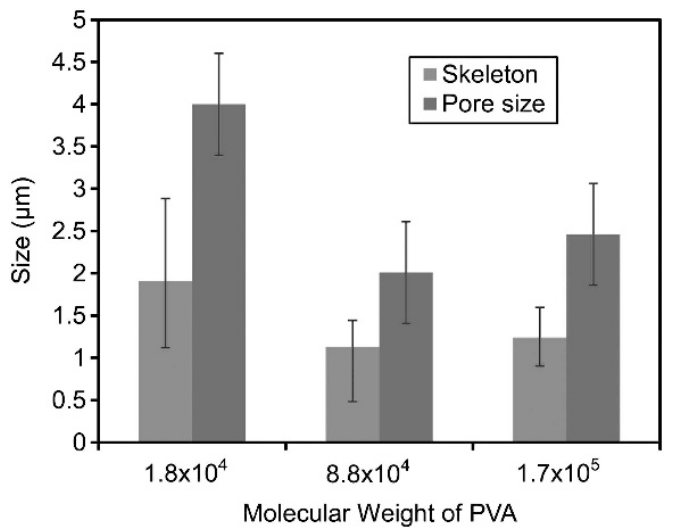

Figure 8 Effect of molecular weight on average pore and skeleton sizes. A full color version of this figure is available at Polymer Journal online.

hydroxyl group of PVA reacts with GA to form the acetal bridge, leading to crosslinking of the PVA chain.

The degree of crosslinking is quantified by the swelling ratio of the crosslinked monolith. In most cases, the swelling equilibrium is 

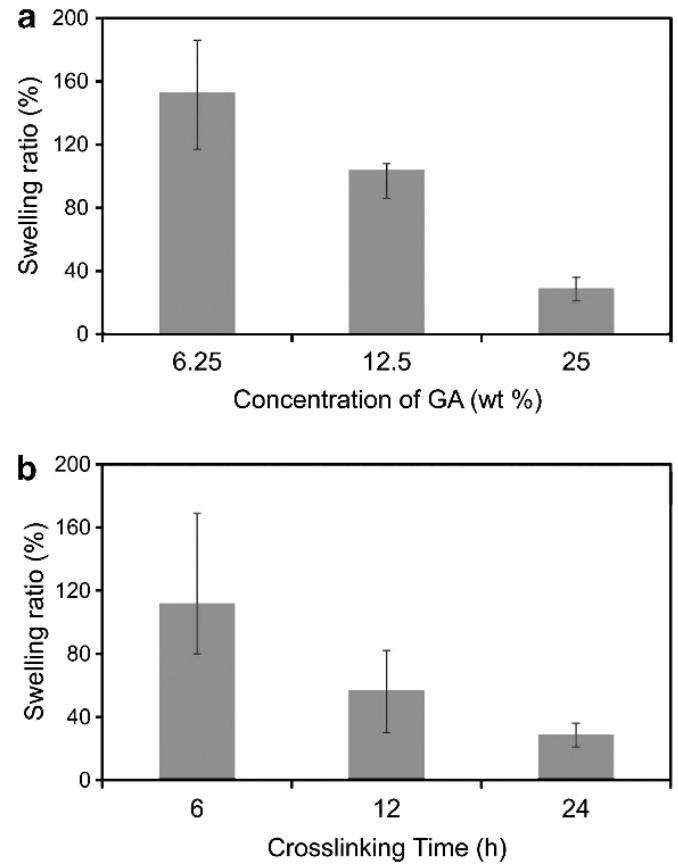

Figure 9 Swelling behavior of the poly(vinyl alcohol) (PVA) monolith with (a) various GA concentrations and (b) various cross-linking times. A full color version of this figure is available at Polymer Journal online.

achieved within $1 \mathrm{~h}$. Figure 9a shows the effect of the GA concentration on the swelling ratio of the sample that is crosslinked for $24 \mathrm{~h}$. As the concentration increases, the swelling ratio decreases, suggesting that the GA solution concentration has an important role in the swelling ratio. The crosslinking period also affects the swelling ratio (Figure 9b). For a GA concentration of 25 wt \%, the swelling ratio decreases as a function of the reaction time. These results suggest that the crosslinking density and swelling ratio of the PVA monolith can be easily tuned by changing the crosslinking conditions.

\section{CONCLUSION}

A new monolith formation method has been developed using TINIPS to produce a hydrophilic monolith. For a water/acetone mixed ratio of $4 / 3$, a PVA monolith with uniform structure and large specific surface area can be formed. By changing the concentration and molecular weight of PVA, as well as the cooling temperature, the skeleton and pore sizes can be easily controlled. Furthermore, the monolith becomes water insoluble by crosslinking with GA. The swelling ratio depends on the GA concentration and the crosslinking time.

To date, most of reported monoliths are composed of hydrophobic polymers. ${ }^{54}$ In this study, a PVA monolith with hydrophilic and biocompatible properties is conveniently fabricated by TINIPS. This process of monolith fabrication, which uses a polymer as the precursor and relies on phase separation techniques (thermally induced phase separation, non-solvent-induced phase separation and TINIPS), may facilitate new applications in various fields. Very recently, both a polycarbonate monolith with a controlled morphology and its blend monolith have been successfully obtained by non-solvent-induced phase separation. ${ }^{55,56}$ Further studies on applications of crosslinked PVA monoliths for bioseparation/ purification are under way in our laboratory.

\section{CONFLICT OF INTEREST}

The authors declare no conflict of interest.

\section{ACKNOWLEDGEMENTS}

This study is financially supported by a Grant-in-Aid for Scientific Research from the Japan Society for the Promotion of Science (no. 21350124).

1 Scheibitz, B., Prager, A. \& Buchmeiser, M. R. Schrock catalyst triggered, ring-opening metathesis polymerization based synthesis of functional monolithic materials. Macromolecules 42, 3493-3499 (2009).

2 Beckert, S., Stallmach, F., Bandari, R. \& Buchmeiser, M. R. Self-diffusion of polystyrene solutions in porous acrylate-based monoliths studied by ${ }^{1} \mathrm{H}$ PFG NMR. Macromolecules 43, 9441-9446 (2010).

3 Rose, M., Klein, N., Senkovska, I., Schrage, C., Wollmann, P., Böhlmann, W., Böhringer, B., Fichtner, S. \& Kaskel, S. A new route to porous monolithic organic frameworks via cyclotrimerization. J. Mater. Chem. 21, 711-716 (2011).

4 Urban, J., Svec, F. \& Fréchet, J. M. J. A monolithic lipase reactor for biodiesel production by transesterification of triacylglycerides into fatty acid methyl esters. Biotechnol. Bioeng. 109, 371-380 (2012).

5 Walsh, Z., Levkin, P. A., Abele, S., Scarmagnani, S., Heger, D., Klán, P., Diamond, D., Paull, B., Svec, F. \& Macka, M. Polymerisation and surface modification of methacrylate monoliths in polyimide channels and polyimide coated capillaries using 660nm light emitting diodes. J. Chromatogr. A 1218, 2954-2962 (2011).

6 Nordborg, A., Hilder, E. F. \& Haddad, P. R. Monolithic phases for ion chromatography. Annu. Rev. Anal. Chem. 4, 197-226 (2011).

7 Peters, E. C., Petro, M., Svec, F. \& Fréchet, J. M. J. Molded rigid polymer monoliths as separation media for capillary electrochromatography. Anal. Chem. 69, 3646-3649 (1997).

8 Eeltink, S., Herrero-Martinez, J. M., Rozing, G. P., Schoenmakers, P. J. \& Kok, W. Th. Tailoring the morphology of methacrylate ester-based monoliths for optimum efficiency in liquid chromatography. Anal. Chem. 77, 7342-7347 (2005).

9 Tao, S., Wang, Y. \& An, Y. Superwetting monolithic $\mathrm{SiO}_{2}$ with hierarchical structure for oil removal. J. Mater. Chem. 21, 11901-11907 (2011).

10 Liu, Y., Ren, L. \& Liu, Z. A unique boronic acid functionalized monolithic capillary for specific capture, separation and immobilization of cis-diol biomoleculesw. Chem. Commun. 47, 5067-5069 (2011).

11 Izquierdo-Barba, I., Vallet-Regí, M., Kupferschmidt, N., Terasaki, O., Schmidtchen, A \& Malmsten, M. Incorporation of antimicrobial compounds in mesoporous silica film monolith. Biomaterials 30, 5729-5736 (2009).

12 Worsley, M. A., Stadermann, M., Wang, Y. M., Satcher, Jr J. H. \& Baumann, T. F. High surface area carbon aerogels as porous substrates for direct growth of carbon nanotubes. Chem. Commun. 46, 9253-9255 (2010).

13 Aguado, S., Canivet, J. \& Farrusseng, D. Engineering structured MOF at nano and macroscales for catalysis and separation. J. Mater. Chem. 21, 7582-7588 (2011).

14 Bourret, G. R., Goulet, P. J. G. \& Lennox, R. B. Synthesis of porous metallic monoliths via chemical reduction of $\mathrm{Au}(\mathrm{I})$ and $\mathrm{Ag}(\mathrm{I})$ nanostructured sheets. Chem. Mater. 23, 4954-4959 (2011).

15 Sachse, A., Hulea, V., Finiels, A., Coq, B., Fajula, F. \& Galarneau, A. Alumina-grafted macro-/mesoporous silica monoliths as continuous flow microreactors for the Diels-Alder reaction. J. Catal. 287, 62-67 (2012).

16 Svec, F. Organic polymer monoliths as stationary phases for capillary HPLC. J. Sep. Sci. 27, 1419-1430 (2004).

17 Minakuchi, H., Nakanishi, K., Soga, N., Ishizuka, N. \& Tanaka, N. Octadecylsilylated porous silica rods as separation media for reversed-phase liquid chromatography. Anal. Chem. 68, 3498-3501 (1996).

18 Nordborg, A. \& Hilder, E. F. Recent advances in polymer monoliths for ion-exchange chromatography. Anal Bioanal. Chem. 394, 71-84 (2009).

19 Li, Y., Tolley, H. D. \& Lee, M. L. Poly[hydroxyethyl acrylate-co-poly(ethylene glycol) diacrylate] monolithic column for efficient hydrophobic interaction chromatography of proteins. Anal. Chem. 81, 9416-9424 (2009).

20 Chen, X., Tolley, H. D. \& Lee, M. L. Polymeric cation-exchange monolithic columns containing phosphoric acid functional groups for capillary liquid chromatography of peptides and proteins. J. Chromatogr. A 1217, 3844-3854 (2010).

21 Satterfield, B. C., Stern, S., Caplan, M. R., Hukari, K. W. \& West, J. A. A. Microfluidic purification and preconcentration of mRNA by flow-through polymeric monolith. Anal. Chem. 79, 6230-6235 (2007).

22 Sáfrány, Á., Beiler, B., László, K. \& Svec, F. Control of pore formation in macroporous polymers synthesized by single-step $\gamma$-radiation-initiated polymerization and cross-linking. Polymer 46, 2862-2871 (2005).

23 Sinner, F. \& Buchmeiser, M. R. A new class of continuous polymer supports prepared by ring-opening metathesis polymerization: a straightforward route to functionalized monoliths. Macromolecules 22, 5777-5786 (2000).

24 Sudheendran, M. \& Buchmeiser, M. R. A continuous bioreactor prepared via the immobilization of trypsin on aldehyde-functionalized, ring-opening metathesis polymerization-derived monoliths. Macromolecules 43, 9601-9607 (2010).

25 Hjertén, S., Liao, J. L. \& Zhang, R. High-performance liquid chromatography on continuous polymer beds. J. Chromatogr. 473, 273-275 (1989). 
26 Wang, Q. C., Svec, F. \& Fréchet, J. M. J. Macroporous polymeric stationary-phase rod as continuous separation medium for reversed-phase chromatography. Anal. Chem. 65 2243-2248 (1993).

27 Svec, F. Porous polymer monoliths: amazingly wide variety of techniques enabling their preparation. J. Chromatogr. A 1217, 902-924 (2010).

28 Svec, F. \& Fréchet, J. M. J. Kinetic control of pore formation in macroporous polymers. Formation of 'molded' porous materials with high flow characteristics for separations or catalysis. Chem. Mater. 7, 707-715 (1995).

29 Badaloni, E., Barbarino, M., Cabri, W., D,Acquarica, I., Forte, M., Gasparrini, F., Giorgi, F., Pierini, M., Simone, P., Ursini, O. \& Villani, C. Efficient organic monoliths prepared by $\gamma$-radiation induced polymerization in the evaluation of histone deacetylase inhibitors by capillary(nano)-high performance liquid chromatography and ion trap mass spectrometry. J. Chromatogr. A. 1218, 3862-3875 (2011).

30 Sun, X., Yang, W., Pan, T. \& Woolley, A. T. Affinity monolith-integrated poly(methy methacrylate) microchips for on-line protein extraction and capillary electrophoresis. Anal. Chem. 80, 5126-5134 (2008)

31 Fernández-Trillo, F. Hest, J. C. M. Thies, J. C. Michon, T., Weberskirch, R. \& Cameron, N. R. Reversible immobilization onto PEG-based emulsion-templated porous polymers by co-assembly of stimuli responsive polymers. Adv. Mater. 21, 55-59 (2009).

32 Plieva, F. M., Karlsson, M., Aguilar, M. R., Gomez, D., Mikhalovsky, S. \& Galaev, I. Y. Pore structure in supermacroporous polyacrylamide based cryogels. Soft Matter 1, 303-309 (2005).

33 Kanamori, K., Nakanishi, K. \& Hanada, T. Rigid macroporous poly(divinylbenzene) monoliths with a well-defined bicontinuous morphology prepared by living radical polymerization. Adv. Mater. 18, 2407-2411 (2006).

34 Audouin, F., Birot, M., Pasquinet, É., Deleuze, H., Besnard, O. \& Poullain, D. Synthesis of porous materials by 2-nitroresorcinol/cyanuric chloride thermal polycondensation in emulsions. J. Appl. Polym. Sci. 108, 2808-2813 (2008).

35 Okada, K., Nandi, M., Maruyama, J., Oka, T., Tsujimoto., T., Kondoh, K. \& Uyama, H. Fabrication of mesoporous polymer monolith: a template-free approach. Chem. Commun. 47, 7422-7424 (2001).

36 Nandi, M., Okada, K. \& Uyama, H. Functional mesoporous polymer monolith for application in ion-exchange and catalysis. Funct. Mater. Lett. 4, 407-410 (2011).

37 Yen, C., He, H., Lee, L. J. \& Ho, W. S. W. Synthesis and characterization of nanoporous polycaprolactone membranes via thermally- and nonsolvent-induced phase separations for biomedical device application. J. Membrane Sci. 343, 180-188 (2009).

38 Susanto, H. \& Ulbricht, M. Characteristics, performance and stability of polyethersulfone ultrafiltration membranes prepared by phase separation method using different macromolecular additives. J. Membrane Sci. 327, 125-135 (2009).

39 Hassan, C. M. \& Peppas, N. A. Structure and applications of poly(vinyl alcohol) hydrogels produced by conventional crosslinking or by freezing/thawing methods. Adv. Polym. Sci. 153, 37-65 (2000).

40 Zhu, H., Li, Y., Qiu, R., Shi, L., Wu, W. \& Zhou, S. Responsive fluorescent $\mathrm{Bi}_{2} \mathrm{O}_{3} @ P V A$ hybrid nanogels for temperature-sensing, dual-modal imaging, and drug delivery. Biomaterials 33, 3058-3069 (2012).
41 Lee, H., Mensire, R., Cohen, R. E. \& Rubner, M. F. Strategies for hydrogen bonding based layer-by-layer assembly of poly(vinyl alcohol) with weak polyacids. Macromolecules 45, 347-355 (2012).

42 Zhou, Y., Yang, D., Chen, X., Xu, Q., Lu, F. \& Nie, J. Electrospun water-soluble carboxyethyl chitosan/poly(vinyl alcohol) nanofibrous membrane as potential wound dressing for skin regeneration. Biomacromolecules 9, 349-354 (2008).

43 Zan, X., Kozlov, M., McCarthy, T. J. \& Su, Z. Covalently attached, silver-doped poly(vinyl alcohol) hydrogel films on poly(L-lactic acid). Biomacromolecules 11, 1082-1088 (2010).

44 Holloway, J. L., Spiller, K. L., Lowman, A. M. \& Palmese, G. R. Analysis of the in vitro swelling behavior of poly(vinyl alcohol) hydrogels in osmotic pressure solution for soft tissue replacement. Acta Biomaterialia 7, 2477-2482 (2011).

45 Doria-Serrano, M. C., Ruiz-Treviño, F. A., Rios-Arciga, C., Hernández-Esparza, M. \& Santiago, P. Physical characteristics of poly(vinyl alcohol) and calcium alginate hydrogels for the immobilization of activated sludge. Biomacromolecules $\mathbf{2}$, 568-574 (2001).

46 Bai, X., Ye, Z., Li, Y. \& Ma, Y. Macroporous poly(vinyl alcohol) foam crosslinked with epichlorohydrin for microorganism immobilization. J. Appl. Polym. Sci. 117, 2732-2739 (2010).

47 Sing, K. S. W. Reporting physisorption data for gas/solid systems. Pure Appl. Chem. 54, 2201-2218 (1982)

48 Coleman, N. J. \& Hench, L. L. A gel-derived mesoporous silica reference material for surface analysis by gas sorption 1. Textural features. Ceram. Int. 26 , $171-178$ (2000).

49 Bunn, C. W. Crystal structure of polyvinyl alcohol. Nature 161, 929-930 (1948).

50 Otsuka, E., Sugiyama, M. \& Suzuki, A. Formation and destruction of physical crosslinks by mild treatments in chemically crosslinked poly(vinyl alcohol) gels. Polym. Bull. 67, 1215-1226 (2011).

$51 \mathrm{Du}$, H. \& Zhang, J. Solvent induced shape recovery of shape memory polyme based on chemically cross-linked poly(vinyl alcohol). Soft Matter. 6, 3370-3376 (2010).

52 Mansur, H. S., Oréfice, R. L. \& Mansur, A. A. P. Characterization of poly(vinyl alcohol)/ poly(ethylene glycol) hydrogels and PVA-derived hybrids by small-angle X-ray scattering and FTIR spectroscopy. Polymer 45, 7193-7202 (2004).

53 Mansur, H. S., Sadahira, C. M., Souza, A. N. \& Mansur, A. A. P. FTIR spectroscopy characterization of poly (vinyl alcohol) hydrogel with different hydrolysis degree and chemically crosslinked with glutaraldehyde. Mater. Sci. Eng. C 28, 539-548 (2008).

54 Calleri, E., Ambrosini, S., Temporini, C. \& Massolini, G. New monolithic chromatographic supports for macromolecules immobilization: challenges and opportunities. J Pharmaceut. Biomed. 69, 64-76 (2012).

55 Xin, Y., Fujimoto, T. \& Uyama, H. Facile fabrication of polycarbonate monolith by nonsolvent induced phase separation method. Polymer 53, 2847-2853 (2012).

56 Xin, Y. \& Uyama, H. Fabrication of polycarbonate and poly(3-hydroxybutyrate-co-3hydroxyhexanoate) blend monolith via non-solvent induced phase separation method. Chem. Lett. 41, 1509-1511 (2012).

Supplementary Information accompanies this paper on Polymer Journal website (http://www.nature.com/pj) 